\title{
Country Familiarity: Three Essays on Entrepreneur Foreign Market Selection
}

\section{Daniel Clark}

\section{Ph.D. awarded by Indiana University, USA (November 2017)}

\section{BIG Question:}

"How do decision makers use their country familiarity when making foreign market selection decisions?"

Managers, executives and entrepreneurs make organizational decisions. These decisions are the product of individual cognitive decision-making processes. Whether a decision is made by one individual or by a team of people, whether it is highly scrutinized or made autonomously, or whether it is made analytically or intuitively, the decision was made by rationally bound and cognitively constrained individuals. In particular, the foreign market selection decision is enormously complex; there are innumerable potential critical variables to consider when selecting a host country: government stability, taxation rates, culture, geography, transportation infrastructure, language, and the competitive landscape, to name just a few. Each country considered has its own variables that need to be researched and compared to the firm's needs and expectations. So how do individuals make decisions when facing such complexity?

My three-essay dissertation examines how entrepreneurs engage the cognitive process of country familiarity in foreign market selection. Engaging this tool enables entrepreneurs to reach a reasonable conclusion within a reasonable exertion of cognitive effort.

\section{Paper One: Country Familiarity in the Initial Stage of Foreign Market Selection}

In the first paper, I look at how entrepreneurs use country familiarity. Cognition research has found that individuals have two sets of cognitive processes called System 1 and System 2
(Kahneman, 2003). System 1 processes are rapid, intuitive, use little information, and require little cognitive effort; System 2 processes are slower, analytical, use more information, and require more effort. Country familiarity, defined as the sense of knowing about a country, is a System 1 process.

I theorize that when country familiarity is low, the entrepreneur rapidly dismisses the country and wastes no further effort on it. At increasing levels of country familiarity, the entrepreneur commits additional effort to the recall of information about the country. These intentional recollections come with a confirmation bias; the entrepreneur is more likely to recall information that relates to the acceptability of the country as a destination. However, when country familiarity crosses a tipping point, and the entrepreneur has the sense that the country is very familiar, that familiarity prompts low effort spontaneous recollections. Spontaneous recollections do not have a confirmation bias; thus, the entrepreneur will be more likely to recall information that relates to the unacceptability of the country as a destination. These effects resulted in an inverted-U curvilinear effect of country familiarity on both cognitive effort and their country assessment.

I further investigate the boundary condition of international experience in the context of country familiarity employment in foreign market selection. High international experience reduces the influence of country familiarity as it provides decision makers with a perspective about what they need to know and do not need to know to evaluate the suitability of a country. As such, the entrepreneurs will be more reluctant to dismiss quickly both low and high familiarity countries, and engaged in more intentional recollections for both. Consequently, there was higher cognitive effort and likelihood of country selection at both the low and high levels of country familiarity. Figure 1 provides a visual illustration of my main theoretical arguments. 
Figure 1. Main Theoretical Arguments

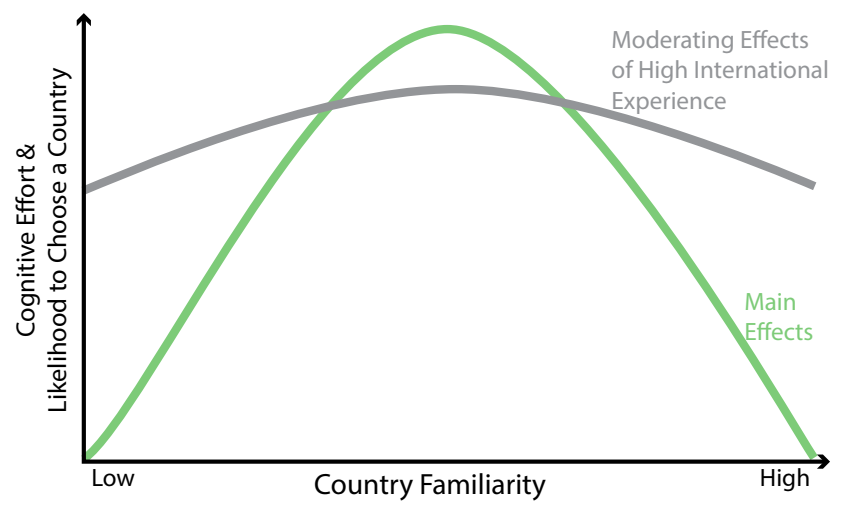

Using verbal protocol analysis, I ran 17 entrepreneurs through a series of 10 foreign market selection decisions to capture their decision-making thought segments, which enabled my analyses of how their country familiarity affected foreign market selection. Ample evidence was detected that entrepreneurs indeed actively employ country familiarity in foreign market selection. Country familiarity allowed the entrepreneurs to assess rapidly how much they thought they knew about each country. It influenced what information they used in the decision, how the decision was made, and ultimately the country they selected. I also found that the entrepreneurs' international experience moderated the effects of country familiarity.

There are several important takeaways from these findings. First, country familiarity is a long-neglected yet important cognitive process useful for understanding individual cognition in foreign market selection. Second, individual cognition, in particular intuitive (not just analytical) decision making, is critical for managing cognitive effort in foreign market selection. Third, international experience plays a meaningful role influencing the use of cognitive processes in foreign market selection. Future foreign market selection research would be well advised to consider individual cognition as a component in decision making, and country familiarity is likely a useful tool for this purpose.

\section{Paper Two: Knowledge Proxies and Internationalization}

One of the interesting components from the previous paper is that country familiarity informs the decision maker about the availability of information without them necessarily accessing the information, and that decisions were made on the strength of the proxy. This means that country familiarity is a knowledge proxy. The internationalization literature (see Clarke, Tamaschke, \& Liesch, 2013; Coviello \& Munro, 1995; Dow, Håkanson, \& Ambos, 2014; Williams \& Grégoire, 2015) has identified other important knowledge proxies, such as psychic distance (indicates the availability of information from external sources), networks (information from connections), homehost similarity (information extrapolated from the home country), and country specific experience (information from personal exposure). In this paper, I conduct a conjoint analysis experiment with 100 entrepreneurs, asking them to make 16 country assessments based on differing levels of each of these five knowledge proxies, to examine the relative value of these knowledge proxies in foreign market selection and their unique contribution in international business research.

Conjoint analysis allowed me to disaggregate and evaluate the unique contribution of each knowledge proxy to foreign market selection decision making. What I found was that entrepreneurs used all of the knowledge proxies. They had no single identifiable preference for one proxy over another; meaning there were no interactions and no obvious "winner". However, there were some differences between the proxies; psychic distance and then country familiarity had the largest influence on specific choices. Beyond the value of each individual proxy, generally, it was more important to have more proxies than specific proxies available; suggesting that beyond the source of information, entrepreneur decision making may be strongly influenced by perception of the quantity of information available.

I also examine how entrepreneurial motivations (entrepreneurial self-efficacy and entrepreneurial drive) moderate how knowledge proxies are used. The first moderator I looked at was entrepreneurial self-efficacy (sometimes called entrepreneurial confidence), the degree to which an entrepreneur believes they will be successful in entrepreneurial activities. Entrepreneurs with high self-efficacy, have the tendency to improvise and to make do with what they have. They tend to terminate information searches earlier than those with lower self-efficacy. As such, those with high self-efficacy were more likely to use and attributed greater importance to each knowledge proxy. The second moderator was entrepreneurial drive. To measure drive, I created a new scale called individual international entrepreneurial orientation (IIEO). To build and validate the scale I followed best practices using three separate samples of students and entrepreneurs. Those with entrepreneurial drive are motivated to take risks, to innovate, and to act proactively; they are also more likely to use the knowledge proxies to make foreign market selection decisions.

This essay delivers several important theoretical contributions. First, country familiarity is useful even in the presence of the more established knowledge proxies. Second, information quantity attracts entrepreneurs, which is somewhat counterintuitive given that entrepreneurs employ country familiarity to reduce cognitive effort, and additional information increases effort-it may be that information is like insurance, entrepreneurs want to know it is there even if they do not want to use 
it. Third, motivations play a key role in how entrepreneurs use knowledge proxies, including country familiarity, in decision making. Finally, I developed a new measure, the IIEO scale, which builds on the established entrepreneurial orientation (Covin \& Wales, 2012) and international entrepreneurial orientation (Covin \& Miller, 2014) literature, and fills the need for an individual-level scale. Interestingly this scale also seemed to predict the likelihood that entrepreneurs would want to internationalize; high IIEO entrepreneurs had higher country assessment scores regardless of the number of knowledge proxies present.

\section{Paper Three: A Multi-Dimensional Perspective of Country Familiarity}

For each of the two previous papers, I considered country familiarity as a single general construct. However, the definition of country familiarity as a sense of knowing about a country raises a natural question: "knowing what?" Research in international business has found that there are many aspects of a country that can influence its attractiveness to potential investors (Nielsen, Asmussen, \& Weatherall, 2017). They are country institutions, culture, industry competition, available products, and customer markets. In this paper, I consider a multi-dimensional view of country familiarity. I examine the relative importance of an entrepreneur's familiarity (sense of knowing) with a county's institutions, culture, industry, products, and markets.

Using the same sample from the previous study, I conducted a second conjoint analysis experiment. I also utilized a unique analytical technique that allows the integration of the information two separate conjoint exercises. In this study, I examined the relative effects of each dimension of country familiarity and examine the potential moderating effects of the entrepreneur's cognitive style; the individual's unique preferences for how they make decisions. I evaluated their cognitive style (either intuitive or analytical) from the prior study's data.

Empirical evidence shows that each of the country familiarity dimensions had a significant positive effect on the country assessments of entrepreneurs. The order of influence (from most to least) was product, market, industry, culture and then institutional familiarity. Because of the direct effect of IIEO in the previous study, I included both entrepreneurial self-efficacy and IIEO as controls in this study. Again, I found a significant direct effect of IIEO on country assessments, providing more evidence of the effect of IIEO on the tendency to internationalize.

The moderator analysis from this paper was particularly interesting. Those with an intuitive cognitive style were more prone to attribute importance to each of the five country familiarity dimensions, which is logical, as an intuitive person is more likely to rely on their intuition. In comparison, entrepreneurs with an analytical cognitive style used country familiarity dimensions more strategically. These entrepreneurs were more likely to use their familiarity when information was particularly expensive, difficult to obtain, or potentially unreliable, specifically their product and market familiarity. There was no effect of analytical cognitive style on either institutional, cultural or industry familiarity.

The first key takeaway from these findings is that country familiarity is a multi-dimensional construct, and is useful both when considered as a single general construct or through its separate dimensions. However, we still do not know much about the relationship between country familiarity and its dimensions. Second, entrepreneurs consider familiarity with institutions, culture, industry, products and markets important in foreign market selection; the absence of any of these can affect the likelihood of country selection. Third, cognitive style matters. Individuals use familiarity differently based on their decision-making preferences. People that are more intuitive are more likely to rely on their familiarity, while more analytical people will selectively apply familiarity to supplement or compensate for other types of information.

\section{Summary of the Dissertation"s Contributions to Theory and Practice}

Beyond the contributions of the individual papers described above, my dissertation makes additional theoretical contributions. First, I introduce country familiarity to the literature in foreign market selection. I repeatedly show that country familiarity, which can be either a general or a multi-dimensional construct, influences foreign market selection decision making. It plays a key role in helping decision makers to manage their cognitive effort, but also influences what decisions they make, reinforcing the importance of understanding individual cognition in foreign market selection. Second, decision makers use knowledge proxies in the place of information to help reduce cognitive effort. Knowing that information is available if needed provides the decision maker freedom to make choices without the cognitively effortful processes of searching for and sorting information. Country familiarity is one knowledge proxy, but there are many others. Third, I introduce to foreign market selection research two useful methodologies: IIEO and the iterative conjoint analysis technique. IIEO will help future researchers measure an individual's drive to internationalize, and the iterative conjoint analysis technique allows researchers to consider decision-making sequence when using conjoint analysis.

My dissertation also makes important practical contributions. It is important for entrepreneurs to understand the effect that country familiarity has on their decision making. We all use 
System 1 tools, and we trust them, but they do affect the choices we make. Research has shown that individuals can opt to use analytical processes to check and backstop their intuitive processes (Kahneman, 2003). The more comfortable an entrepreneur is with intuitive decision making, the more important it is for them to consider the potential biases that country familiarity and other System 1 processes have. Using analytical processes selectively, to confirm their decision making, does not need to dramatically increase decision effort. Another option is to involve others (coworkers, friends, or network links) in the decision and allow them to provide a "sober second thought" to the primary decision maker. The lesson here is not "don't trust country familiarity," which is neither accurate nor practical. Country familiarity is useful because it works, and research has shown that System 1 decision making can be very accurate (Gigerenzer \& Gaissmaier, 2011). However, foreign market selection decision making is both complex and risky, and for those particularly prone to favoring one cognitive process, verifying the decision is simply prudent. This is particularly true for confident and driven entrepreneurs. The more entrepreneurs trust themselves and their abilities and the more that they "want" to pursue international opportunities; the more likely they are to use country familiarity to help them make their decisions.

Country familiarity is unique in that it is both an important component of foreign market selection cognition, and is easily accessible by both decision makers and researchers. One can assess their own country familiarity at any time, and can provide that information to others with ease. That ease is what makes country familiarity so influential; it is effectively the most accessible foreign market selection tool. The fact that entrepreneurs, and likely other managers, use country familiarity is not surprising, but its effect on decision making and its apparent centrality to the foreign market selection process is surprising.

\section{References}

Clark, D. R., Li, D., \& Shepherd, D. A. 2018. Country familiarity in the initial stage of foreign market selection. Journal of International Business Studies, 49(4): 442-472.

Clarke, J. E., Tamaschke, R., \& Liesch, P. W. 2013. International experience in international business research: A conceptualization and exploration of key themes. International Journal of Management Reviews, 15(3): 265-279.

Coviello, N. E., \& Munro, H. J. 1995. Growing the entrepreneurial firm: Networking for international market development. European Journal of Marketing, 29(7): 49-61.

Covin, J. G., \& Miller, D. 2014. International entrepreneurial orientation: Conceptual considerations, research themes, measurement issues, and future research directions. Entrepreneurship: Theory \& Practice, 38(1): $11-44$.

Covin, J. G., \& Wales, W. J. 2012. The measurement of entrepreneurial orientation. Entrepreneurship: Theory \& Practice, 36(4): 677-702.
Dow, D., Håkanson, L., \& Ambos, B. 2014. Perceptions versus national-level differences: A mediating model of psychic distance. In A. Verbeke, R. Van Tulder, \& S. Lundan (Eds), Multinational Enterprises, Markets and Institutional Diversity. Bingley, UK: Emerald Group Publishing Limited.

Gigerenzer, G., \& Gaissmaier, W. 2011 . Heuristic decision making. Annual Review of Psychology, 62: 451-482.

Kahneman, D. 2003. A perspective on judgment and choice: Mapping bounded rationality. American Psychologist, 58(9): 697-720.

Maitland, E., \& Sammartino, A. 2015. Managerial cognition and internationalization. Journal of International Business Studies, 46(7): 733-760.

Nelson, R. R., \& Winter, S. G. 2002. Evolutionary theorizing in economics. Journal of Economic Perspectives, 16(2): 23-46.

Nielsen, B. B., Asmussen, C. G., \& Weatherall, C. D. 2017. The location choice of foreign direct investments: Empirical evidence and methodological challenges. Journal of World Business, 52(1): 62-82.

Williams, D. W., \& Grégoire, D. A. 2015. Seeking commonalities or avoiding differences: Re-conceptualizing distance and its effects on internationalization decisions. Journal of International Business Studies, 46(3): 253-284.

Daniel Clark (daniel.clark@ie.edu) is an assistant professor of Entrepreneurship at IE Business School in Madrid, Spain. He earned his Ph.D. from Indiana University's Kelley School of Business. His research lies at the intersection of entrepreneurship, international business and cognition. He is specifically interested in examining how entrepreneurs make complex decisions such as internationalization, and the idiosyncrasies in their use of cognition processes in decision making. 